\title{
IMPACTO DE LAS ACTITUDES DE LOS RESIDENTES SOBRE EL GRADO DE ACEPTACIÓN DEL TURISMO DE PLAYA, EL TURISMO DE DEPORTES, EL TURISMO DE NATURALEZA Y EL TURISMO CULTURAL
}

\section{RESUMEN}

\author{
1 María Dolores Sánchez-Fernández \\ ${ }^{2}$ José Ramón Cardona
}

El estudio de las actitudes de los residentes es importante para la gestión de los destinos turísticos. Los residentes forman su actitud hacia el turismo mediante un análisis coste-beneficios basado en su percepción de los impactos del turismo. Los residentes tienen distintos niveles de aceptación de los distintos tipos de oferta turística y el objetivo de este trabajo es contrastar si existe relación entre la actitud hacia el turismo y el grado de aceptación del turismo de playa, el turismo de deportes, el turismo de naturaleza y el turismo cultural. El análisis de los datos se realizó mediante Mínimos Cuadrados Parciales sobre una muestra de 418 residentes de Ibiza, encontrando que: no hay relación causal entre la actitud de los residentes y el grado de aceptación del turismo cultural; las relaciones causales más fuertes son entre la actitud general de los residentes y el turismo de playa y entre la actitud general y el turismo deportivo; la percepción de depender económicamente del turismo tiene efecto significativo sobre el turismo de playa y el turismo deportivo. Parece que el grado de aceptación del turismo deportivo se basa en el análisis crítico de los residentes más que en otras ofertas y su desarrollo estaría acompañado de una mejora en su grado de aceptación.

Palabras Clave: Turismo, Playa, Deporte, Naturaleza, Cultura, Actitudes, Residentes

\section{IMPACT OF RESIDENTS ATTITUDES ON THE ACCEPTANCE DEGREE OF BEACH TOURISM, SPORTS TOURISM, NATURE TOURISM AND CULTURAL TOURISM}

\begin{abstract}
The study of residents' attitudes is important for the management of tourist destinations. Residents formed their attitude towards tourism through a cost-benefits analysis based on their perception of the tourism impacts. Residents have different levels of acceptance of different types of tourism and the aim of this paper is to contrast if there is a relationship between the attitude towards tourism and the degree of acceptance of beach tourism, sports tourism, nature tourism and cultural tourism. The data analysis was performed using Partial Least Squares on a sample of 418 residents of Ibiza, finding that: there is no causal relationship between the attitude of residents and the degree of acceptance of cultural tourism; the strongest causal relationships are between the general attitude of residents and beach tourism and between the general attitude and sports tourism. The main contribution is that a tourism-friendly attitude does not imply an equivalent support to any type of tourism, although not to generate controversy. If there is a strong relationship between general attitude and acceptance of sports tourism, in the case of Ibiza.
\end{abstract}

Keywords: Tourism, Beach, Sport, Nature, Culture, Attitudes, residents

\footnotetext{
${ }^{1}$ Doutora em Programa Oficial de Doutoramento Competitividad, In. pela Universidade da Coruña, UDC, (Espanha). Professora Adjunto da Universidade da Corunã. E-mail: msanchezf@udc.es

${ }^{2}$ Universitat de les Illes Balears, España. Investigador Universitat de les Illes Balears, (España). E-mail: jramon.cardona@terra.com
} 


\section{INTRODUCCIÓN}

Los gestores del sector turístico deben tener en cuenta las actitudes de los residentes si desean un desarrollo turístico sostenible (Ap \& Crompton, 1998). Desde finales de los setenta, se han realizado bastantes estudios acerca de las actitudes de los residentes hacia el turismo (Almeida, Balbuena \& Cortés, 2015; Sharpley, 2014). En un principio los investigadores centraron sus investigaciones en medir actitudes y sus relaciones con los impactos percibidos (Akis, Peristianis \& Warner, 1996; Faulkner \& Tideswell, 1997; Haralambopoulos \& Pizam, 1996; Jurowski, Uysal \& Williams, 1997; Lindberg \& Johnson, 1997). En estas investigaciones se alcanzó un cierto consenso que agrupaba los impactos, beneficios y costes, en tres o cuatro categorías que englobaban el marco económico, social, cultural y medioambiental (Andereck, Valentine, Knopf \& Vogt, 2005; Ayres, 2000; Gursoy \& Rutherford, 2004; Gursoy, Jurowski \& Uysal, 2002). Posteriormente, la literatura inició la búsqueda de variantes que mejoraran la medición de los impactos, beneficios y costes, y por tanto mejoraran la medición de las actitudes. En estas investigaciones se ahondaba en los factores o condicionantes que varían la intensidad o sentido del impacto (Bramwell, 2003; Bujosa \& Rosselló, 2007; Gursoy \& Kendall, 2006; Kim \& Petrick, 2005; Lee \& Back, 2006; Lepp, 2007; Lindberg, Dellaert \& Rassing, 1999; Mackay \& Campbell, 2004; Mason \& Cheyne, 2000; Weaver \& Lawton, 2001; Williams \& Lawson, 2001).

Por otra parte, los diferentes tipos y formas de turismo influyen en las actitudes de los residentes (Murphy, 1985; Williams \& Lawson, 2001) al implicar distintos impactos sobre el entorno local y distintas interacciones entre turistas y residentes. Pero la inmensa mayoría de los estudios de actitudes de los residentes analizan el sector turístico en su conjunto, siendo pocos los casos que se centran en algún tipo concreto de turismo o actividad. La única excepción se produce en los destinos con una elevada especialización en el juego y los casinos (Ko \& Stewart, 2002; Lee, Kim \& Kang, 2003; Vong, 2009; Vong \& McCartney, 2005).

El objetivo de este estudio es determinar cómo afectan las actitudes de los residentes en el grado de aceptación de diversos tipos de turismo, para el caso de la isla de Ibiza. Ibiza es una isla de $572 \mathrm{~km}^{2} \mathrm{de}$ superficie y más de 140.000 habitantes (Ibestat), caracterizada por su fuerte dependencia económica del turismo. Cada año recibe entre $1,700.000$ y 2,000.000 turistas, mayoritariamente concentrados en los meses de junio a septiembre. El turismo predominante en la isla es el de sol y playa (Agència de Turisme de les Illes Balears, 2015). Los tipos de turismo analizados en este estudio son el turismo deportivo, resaltando el cicloturismo y el turismo náutico, y el turismo de naturaleza, especialmente el agroturismo y las actividades en la naturaleza (excursiones, avistamiento de animales, etc.). Como elemento de comparación se añadieron el turismo de sol y playa, al ser la principal oferta de la isla, y el turismo cultural, por existir patrimonio reconocido por la UNESCO en la isla (Ramón, Azpelicueta \& Serra, 2015).

El análisis de los datos se realizó mediante regresión de Mínimos Cuadrados Parciales, en concreto mediante el software estadístico SmartPLS 2.0M3 (Ringle, Wende \& Will, 2005). El interés del estudio radica en analizar si existe relación entre las actitudes de los residentes hacia el turismo en general y su grado de aceptación de los distintos tipos de turismo planteados. Los resultados obtenidos muestran que existe 
una relación causal entre la actitud general de los residentes hacia el turismo y el grado de aceptación de la mayoría de las ofertas planteadas. Pero cuando estas ofertas son tomadas como variables dependientes del modelo, en sustitución de la actitud general hacia el turismo, las relaciones causales son no significativas en su casi totalidad. Por tanto, debe considerarse el apoyo a las distintas formas de turismo una consecuencia de la valoración general del turismo y no de la percepción de los impactos generados por el sector. Cabe indicar que el turismo deportivo obtiene resultados similares al turismo de sol y playa y mejores que el turismo basado en la naturaleza y los espacios rurales.

\section{REVISIÓN DE LA LITERATURA}

Los estudios sobre actitudes de los residentes frente al turismo dividen los impactos percibidos en tres categorías básicas de costes y beneficios: económicos, medioambientales y sociales (Gee, Mackens \& Choy, 1989; McIntosh \& Goeldner, 1990). Diversos estudios sugieren que habitualmente el apoyo al turismo es una estrategia de desarrollo económico de la comunidad local (Brayley, Var \& Sheldon, 1990; Gursoy et al., 2002; Jurowski et al., 1997; Keogh, 1990; Lankford \& Howard, 1994; Liu \& Var, 1986; Prentice, 1993; Teye, Sirakaya \& Sönmez, 2002; Um \& Crompton, 1987) siendo este hecho lo que induce a pensar que la división más adecuada es entre impactos económicos y el resto de impactos. El desarrollo turístico se justifica habitualmente en base a una relación costebeneficio, en particular beneficios económicos versus costes socioculturales y medioambientales (Ayres, 2000; Liu \& Var, 1986; Perdue, Long \& Kang, 1995).
Muchos estudios realizados sobre las actitudes de los residentes incluyen factores económicos (Besculides, Lee \& McCormick, 2002; Bruner, 1996; Gursoy et al., 2002; Jurowski et al., 1997; Liu \& Var, 1986; Madrigal, 1993; Milman \& Pizam, 1988; Teye et al., 2002), principalmente el aumento de las oportunidades de empleo (Besculides et al., 2002; Bruner, 1996; Gursoy et al., 2002; Teye et al., 2002; Var, Kendall \& Tarakcoglu, 1985) y las rentas públicas generadas por el turismo (Gursoy et al., 2002; Jurowski et al., 1997). Los beneficios económicos derivados del turismo tienen un peso muy importante en las actitudes de los residentes (Ap, 1992; Lawson, Williams, Young \& Cossens, 1998; Liu \& Var, 1986; Prentice, 1993), y es el beneficio más importante para la población local al plantear un posible desarrollo turístico (Gursoy et al., 2002). En base a la bibliografía es razonable proponer la hipótesis 1:

Hipótesis 1: La percepción de los beneficios económicos (oportunidades de empleo, inversión y empresariales) generados por el turismo tiene un efecto positivo sobre la actitud general hacia el turismo.

Entre los beneficios no directamente económicos se pueden mencionar las mejoras en las infraestructuras (Liu \& Var, 1986; Milman \& Pizam, 1988) y el aumento de la oferta comercial y de ocio (Liu \& Var, 1986). El turismo conlleva la creación de una potente infraestructura de transportes, suministros y saneamiento para atender a los turistas (Liu \& Var, 1986; Milman \& Pizam, 1988). La accesibilidad a estos recursos e infraestructuras puede influir en las actitudes de los residentes (Gursoy et al., 2002; Keogh, 1990; Perdue, Long \& Allen, 1987), y para Lankford y Howard (1994) es el elemento con más capacidad de predicción de las actitudes de los residentes, siendo razonable proponer la hipótesis 2 :

Hipótesis 2: La percepción de otros beneficios (mejoras en los servicios 
públicos) generados por el turismo tiene un efecto positivo sobre la actitud general hacia el turismo.

Cabe indicar que una mejora económica entre los residentes genera mayores ingresos públicos con los que financiar los servicios públicos y la construcción de infraestructuras diversas (Liu \& Var, 1986; Milman \& Pizam, 1988), mejorando el entorno urbano de los residentes. Por tanto es plausible proponer la hipótesis 3:

Hipótesis 3: La percepción de los beneficios económicos generados por el turismo tiene un efecto positivo sobre la percepción de otros beneficios generados por el turismo.

Como contrapartida de los beneficios, los costes percibidos afectan de forma negativa las actitudes hacia el desarrollo turístico (Jurowski et al., 1997; Keogh, 1990; Long, Perdue \& Allen, 1990; Milman \& Pizam, 1988; Prentice, 1993). La percepción de los costes es, junto a la percepción de los beneficios, uno de los elementos que permite a los residentes realizar una valoración global del turismo, y esta determina su actitud hacia el sector. Todo ello lleva a proponer la hipótesis 4:

Hipótesis 4: La percepción de los costes generados por el turismo tiene un efecto negativo sobre la actitud general hacia el turismo.

Muchas regiones con escasas fuentes de recursos adoptan el turismo como una solución a sus problemas de desarrollo económico (Cater, 1987) y los beneficios económicos previstos hacen que la reacción de la población local sea muy favorable al turismo (Gursoy et al., 2002; Haralambopoulos \& Pizam, 1996). Diversos autores han llegado a la conclusión de que los residentes infravaloran los costes y sobrevaloran los beneficios (Besculides et al., 2002; Liu \& Var, 1986) cuando se encuentran en una mala situación económica. Por tanto cuanto más negativa sea percibida la situación económica local, más entusiasta será la reacción de la población hacia el desarrollo turístico (Gursoy et al., 2002). Finalmente indicar que la dependencia económica tiene una gran influencia en las percepciones y actitudes de los residentes hacia el turismo (Allen, Hafer, Long \& Perdue, 1993; Haralambopoulos \& Pizam, 1996; Johnson, Snepenger \& Akis, 1994; Jurowski et al., 1997; King, Pizam \& Milman, 1993). Todo ello lleva a proponer la hipótesis 5:

Hipótesis 5: La percepción de que Ibiza depende del turismo mejora las actitudes de los residentes hacia el turismo.

Hipótesis 5.1: La percepción de que Ibiza depende del turismo tiene un efecto positivo sobre la percepción de los beneficios económicos generados por el turismo.

Hipótesis 5.2: La percepción de que Ibiza depende del turismo tiene un efecto positivo sobre la percepción de los otros beneficios generados por el turismo.

Hipótesis 5.3: La percepción de que Ibiza depende del turismo tiene un efecto negativo sobre la percepción de los costes generados por el turismo.

Hipótesis 5.4: La percepción de que Ibiza depende del turismo tiene un efecto positivo sobre la actitud general hacia el turismo.

Los estudios sobre la percepción de los impactos del turismo sobre el medioambiente indican una relación positiva (Liu \& Var, 1986; Perdue et al., 1987) o negativa (Liu, Sheldon \& Var, 1987) sobre las actitudes. Una posible explicación de la disparidad de resultados estaría en la existencia de distintas actitudes acerca de la relación entre los seres humanos y el medioambiente (Gursoy et al., 2002), pero es previsible que predomine un efecto negativo sobre los beneficios y positivo sobre los costes. Todo ello lleva a proponer la hipótesis 6: 
Hipótesis 6: La preocupación por el medioambiente empeora las actitudes de los residentes hacia el turismo.

Hipótesis 6.1: La preocupación por el medioambiente tiene un efecto negativo sobre la percepción de los beneficios económicos generados por el turismo.

Hipótesis 6.2: La preocupación por el medioambiente tiene un efecto negativo sobre la percepción de los otros beneficios generados por el turismo.

Hipótesis 6.3: La preocupación por el medioambiente tiene un efecto positivo sobre la percepción de los costes generados por el turismo.

Hipótesis 6.4: La preocupación por el medioambiente tiene un efecto negativo sobre la actitud general hacia el turismo.

Las relaciones causales expuestas en las hipótesis 1 a 6 ya fueron analizadas en Ramón (2015) pero aún no se ha planteado qué efecto puede tener la "Actitud General hacia el Turismo" sobre el grado de aceptación de los diversos tipos de turismo. Al estudiar los tipos de turismo, deben tenerse en cuenta las diferencias en las actitudes de los turistas en el destino, ya que es lo que afecta de forma más significativa a las actitudes de los residentes. No es lo mismo un turista que muestra respeto e interés por la cultura y la sociedad local que un turista que sólo busca libertad a bajo precio y en un ambiente similar a su lugar de origen.

Para los destinos situados en el mar Mediterráneo, Lanquar (2001) perfila cuatro tipos de turistas según sus actitudes y actividades en el destino: el "sedentario", el "sedentario-móvil", el "itinerante" y el "nómada". Otra tipología psicográfica de los turistas fue propuesta por Bigné, Font y Andreu (2000): los "extremo impulsores", los "de intereses especiales", los "intermedios", los "extremo conformistas". Según el destino por el que optan los turistas, Plog (1974) establece una tipología con los alocéntricos (buscan lugares desconocidos sin desarrollo turístico) y los psicocéntricos (huyen de lugares desconocidos y visitan destinos consolidados) en los extremos. Los turistas con motivaciones culturales y aventureras (nómadas, extremo impulsor, alocéntricos, etc.) tienden a mostrar mayor respeto por los residentes y realizan más actividades durante su estancia (Gómez, San Martín \& Bertiche, 1999). En el otro extremo hay los turistas sin ningún interés por la cultura, que buscan descansar y consideran que el destino turístico, residentes incluidos, es quien debe adaptarse a los turistas y no viceversa (sedentarios, extremo conformistas, psicocéntricos, etc.).

Algunos turistas poseen actitudes antiturísticas (Bruckner \& Finkielkraut, 1979; Doran, Larsen \& Wolff, 2015; Gration, Raciti \& Arcodia, 2011; Gustafson, 2002; Jacobsen, 2000; Yu, Kim, Chen \& Schwartz, 2012) y no quieren mezclarse con las masas de turistas, es decir quieren vivir una experiencia original y no preparada para ser vivida por grandes masas de turistas, ya que no se consideran turistas (Hennig, 1997). Las actitudes antiturísticas se han convertido en un símbolo de prestigio ya que con ellas se busca no ser un turista ordinario (Bruckner \& Finkielkraut, 1979). Estas actitudes son una acentuación del individualismo y tiene elementos en común con los turistas alocéntricos, como son el espíritu aventurero, el gusto por la libertad que da el hecho de viajar de forma autónoma (Plog, 1974, 2002).

El tipo e intensidad de los contactos entre visitantes y residentes son variables importantes en la determinación de las actitudes de los residentes (Pearce, 1996), sobre todo cuando el residente trabaja o depende económicamente del turismo (Milman \& Pizam, 1988). La interacción entre la población residente y los visitantes es uno de los factores más importante tanto para determinar las percepciones y actitudes de los residentes (Murphy, 1985), como 
para determinar los niveles de satisfacción de los turistas a través de la percepción que obtienen del destino turístico (Gómez et al., 1999; Nyaupane, Teye \& Paris, 2008; Yu \& Lee, 2014).

El grado de aceptación de un tipo de turismo por parte de los residentes depende de los beneficios y costes que genere y de la actitud de los turistas hacia el destino visitado. Es de esperar que los residentes con actitudes más positivas hacia el turismo posean niveles mayores de aceptación de los diversos tipos de oferta turística y puede proponerse la Hipótesis 7:

Hipótesis 7: La actitud general hacia el turismo mejora el grado de aceptación de las diferentes ofertas turísticas.

En Ibiza, al igual que en el conjunto de Baleares, el turismo de sol y playa familiar es la principal oferta y la única que actualmente puede atraer el elevado volumen de turistas necesario para mantener los ingresos del sector.

\section{Hipótesis 7.1: La actitud general}

hacia el turismo mejora el grado de aceptación del turismo de sol y playa.

El turismo deportivo siempre ha resultado atractivo y las dos líneas principales que se ha buscado desarrollar son el turismo náutico y el cicloturismo. El turismo náutico es una oferta lógica tratándose de una isla. Además, la práctica de la vela ha sido ampliamente potenciada en Baleares por la Familia Real española y por eventos como la Copa del Rey de Vela, celebrada desde 1982 en Palma de Mallorca, y la Ruta de la Sal, con destino en el puerto de Ibiza y celebrada desde 1989. El atractivo de las competiciones de vela favoreció la llegada de yates y en la actualidad estas embarcaciones son plataformas de ocio acuático para el esparcimiento de sus ocupantes. Desde hace años, el cicloturismo se ha intentado potenciar (Garau, Serra \& Tur, 1999a) mediante la creación de rutas ciclistas y carriles especiales. El objetivo pretendido es que las islas Baleares se conviertan en un lugar de entrenamiento para los equipos ciclistas durante el invierno, pero hasta el momento sólo se ha conseguido potenciar esta actividad entre los aficionados locales. El turismo de golf fue estudiado en su momento (Garau, Serra \& Tur, 1997) pero las críticas de importantes sectores de la población residente han frenado totalmente la potenciación de este tipo de turismo.

Hipótesis 7.2: La actitud general

hacia el turismo mejora el grado de aceptación del turismo deportivo.

El turismo en la naturaleza y el espacio rural también se ha intentado potenciar por parte de las administraciones. A partir de 1993 se desarrollo el turismo rural en Baleares (Garau, 2002) con la finalidad de conservar las actividades tradicionales del espacio rural e introducir a los turistas en ellas. Pero en la práctica se ha convertido en una oferta de pequeños hoteles de lujo con poca conexión con las tradiciones locales (Serra, Ramón \& Estades, 2015). Una oferta complementaria del turismo rural es el turismo activo basado en el entorno natural (Garau, Serra \& Tur, 1999b): excursiones, senderismo, buceo, etc. Por el momento la oferta de actividades en el entorno natural no es muy abundante y complementa a otras ofertas.

Hipótesis 7.3: La actitud general

hacia el turismo mejora el grado de aceptación del turismo en la naturaleza.

Finalmente, cabe mencionar el potencial que posee el turismo cultural, especialmente desde que la ciudad histórica y diversos emplazamientos arqueológicos de Ibiza fueron declarados Patrimonio de la Humanidad por la UNESCO (Ramón et al., 2015). Pero en la actualidad su desarrollo es bajo y es una actividad alternativa para los días lluviosos.

Hipótesis 7.4: La actitud general hacia el turismo mejora el grado de aceptación del turismo cultural. 
El modelo causal propuesto en base a las siete hipótesis planteadas es el que se muestra en la Figura 1, y que será objeto de análisis para el caso de Ibiza.

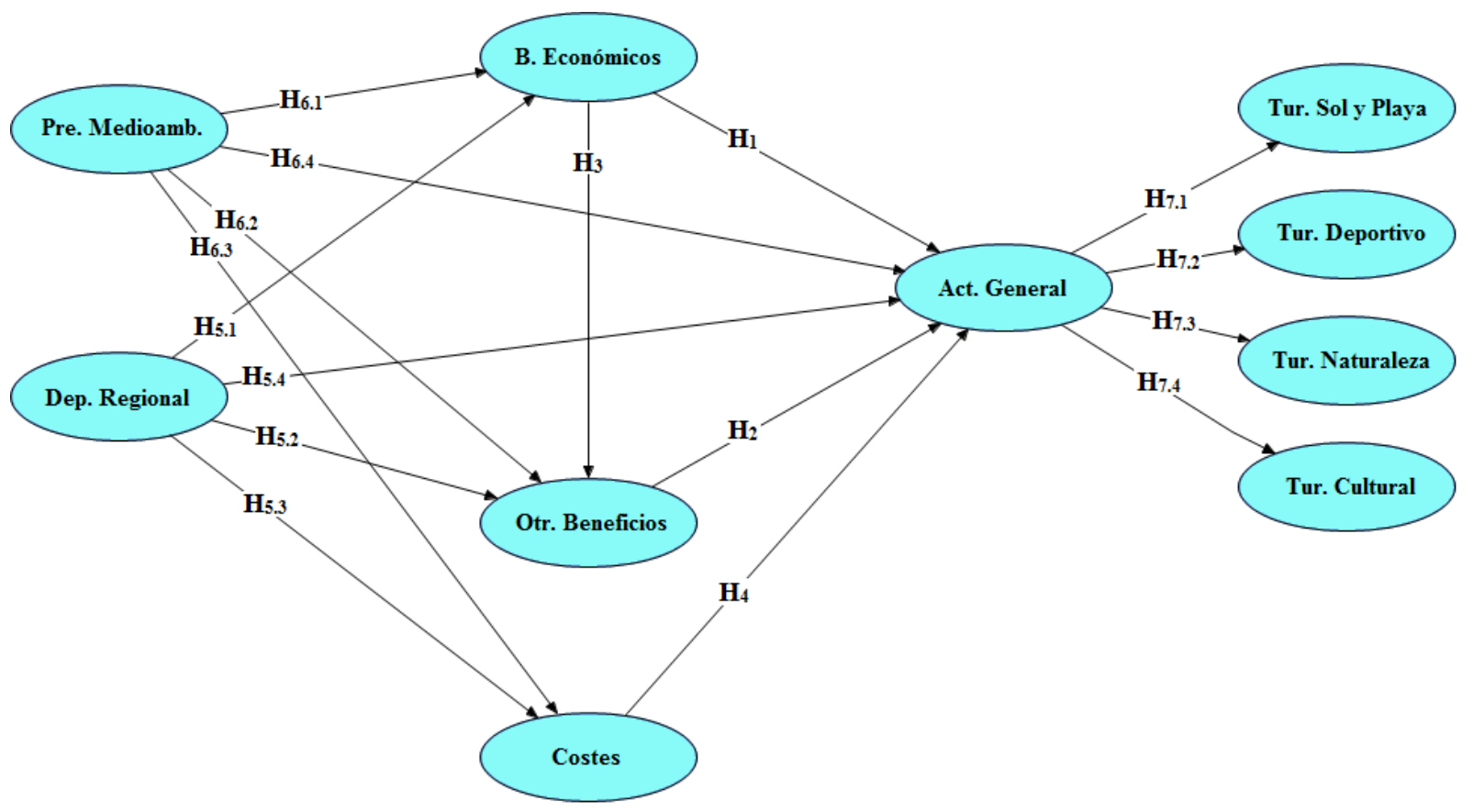

Figura 1. Modelo estructural propuesto.

\section{METODOLOGÍA}

Los datos utilizados proceden de una muestra de residentes de Ibiza obtenida en 2011 mediante un procedimiento aleatorio por el que se seleccionaban calles de cada población y se procedía al reparto de cuestionarios de forma aleatoria dentro de cada calle. Para conseguir el mayor nivel de representatividad posible de la muestra obtenida, se controlaron los parámetros demográficos de los cuestionarios recogidos para solventar posibles sesgos en la composición de la muestra. El cuestionario usado contenía ítems con escalas Likert de cinco puntos como opción de respuesta y un conjunto de preguntas sociodemográficas. Se obtuvieron 418 encuestas válidas con el perfil demográfico indicado en la Tabla 1. El margen máximo de error dado un nivel de confianza del $95 \%$ fue del $4,89 \%$.

Tabla 1. Perfil demográfico de la muestra.

\begin{tabular}{|c|c|c|}
\hline Variable Demográfica & Frec. & $\%$ \\
\hline \multicolumn{3}{|l|}{ Sexo: } \\
\hline Hombre. & 151 & $36,12 \%$ \\
\hline Mujer. & 266 & $63,64 \%$ \\
\hline No contestan. & 1 & $0,24 \%$ \\
\hline \multicolumn{3}{|l|}{ Edad: } \\
\hline Menos de 25. & 55 & $13,16 \%$ \\
\hline
\end{tabular}


Impacto de Las Actitudes de Los Residentes sobre El Grado de Aceptación Del Turismo de Playa, El

Turismo de Deportes, El Turismo de Naturaleza Y El Turismo Cultural

\begin{tabular}{|c|c|c|}
\hline $25-34$ & 102 & $24,40 \%$ \\
\hline $35-44$ & 98 & $23,44 \%$ \\
\hline $45-54$. & 88 & $21,05 \%$ \\
\hline 55 o más. & 48 & $11,48 \%$ \\
\hline No contestan. & 27 & $6,46 \%$ \\
\hline \multicolumn{3}{|l|}{ Nivel de Estudios: } \\
\hline Estudios Primarios. & 92 & $22,01 \%$ \\
\hline Estudios Secundarios. & 175 & $41,87 \%$ \\
\hline Estudios Universitarios. & 148 & $35,41 \%$ \\
\hline No contestan. & 3 & $0,72 \%$ \\
\hline \multicolumn{3}{|l|}{ Años residiendo en la Isla: } \\
\hline Toda la Vida. & 267 & $63,88 \%$ \\
\hline Vine a vivir hace: & 151 & $36,12 \%$ \\
\hline Menos de 5 años. & 21 & $5,02 \%$ \\
\hline 5-14 años. & 47 & $11,24 \%$ \\
\hline 15-24 años. & 20 & $4,78 \%$ \\
\hline 25-34 años. & 41 & $9,81 \%$ \\
\hline 35 o más años. & 20 & $4,78 \%$ \\
\hline \multicolumn{3}{|l|}{ Trabajan en el sector turístico: } \\
\hline Si. & 256 & $61,24 \%$ \\
\hline No. & 157 & $37,56 \%$ \\
\hline No contestan. & 5 & $1,20 \%$ \\
\hline \multicolumn{3}{|l|}{ Ingresos netos familiares anuales: } \\
\hline Menos de $15.000 €$ & 46 & $11,00 \%$ \\
\hline De $15.001 €$ a $30.000 €$ & 150 & $35,89 \%$ \\
\hline De $30.001 €$ a $45.000 €$ & 96 & $22,97 \%$ \\
\hline De $45.001 €$ a $60.000 €$ & 37 & $8,85 \%$ \\
\hline De $60.001 €$ a $75.000 €$ & 12 & $2,87 \%$ \\
\hline Más de $75.000 €$ & 10 & $2,39 \%$ \\
\hline No contestan. & 67 & $16,03 \%$ \\
\hline \multicolumn{3}{|c|}{ Efecto de una crisis turística en los ingresos familiares: } \\
\hline Bajarían mucho. & 161 & $38,52 \%$ \\
\hline Bajarían. & 170 & $40,67 \%$ \\
\hline No cambiarían. & 84 & $20,10 \%$ \\
\hline No contestan & 3 & $0,72 \%$ \\
\hline
\end{tabular}

Fuente: elaboración propia.

Para la realización del análisis causal se utilizaron un conjunto de ítems cuya posibilidad de respuesta consistía en escalas Likert de cinco puntos que medían el grado de acuerdo con la afirmación propuesta, siendo 1 "Muy en Desacuerdo", 3 "Indiferente" y 5 "Muy de Acuerdo", en el caso de los constructos "Beneficios Económicos", "Otros Beneficios", "Costes", "Dependencia Regional", "Preocupación por el Medioambiente" y "Actitud General hacia el Turismo". Y en el caso de los constructos "Turismo de Sol y Playa", "Turismo Deportivo", "Turismo en la Naturaleza" y "Turismo Cultural" la escala Likert medía el grado de aceptación del tipo de oferta turística, siendo 1 "Totalmente Inaceptable", 3 "Indiferente" y 5 "Totalmente Aceptable". A los datos correspondientes a los tipos de oferta turística se les realizó un primer análisis descriptivo (Tabla 2) mediante el software estadístico DYANE 4.0 (Santesmases, 2009), no obteniendo diferencias estadísticamente significativas por municipios en base a un análisis de varianza realizado mediante la $\mathrm{F}$ de Snedecor. 
Tabla 2. Grado de aceptación de las ofertas turísticas analizadas.

\begin{tabular}{|l|c|c|c|c|c|c|}
\hline \multirow{2}{*}{ Denominación } & \multirow{5}{*}{} & \multicolumn{5}{|c|}{ Municipio de Residencia } \\
\cline { 3 - 7 } & Total & Eivissa & $\begin{array}{c}\text { Sant } \\
\text { Antoni }\end{array}$ & $\begin{array}{c}\text { Sant } \\
\text { Joan }\end{array}$ & $\begin{array}{c}\text { Sant } \\
\text { Josep }\end{array}$ & $\begin{array}{c}\text { Santa } \\
\text { Eulària }\end{array}$ \\
\hline Tamaño de la muestra: & $\mathbf{4 1 8}$ & $\mathbf{1 4 1}$ & $\mathbf{8 0}$ & $\mathbf{1 7}$ & $\mathbf{7 3}$ & $\mathbf{1 0 7}$ \\
\hline Turismo de Sol y Playa: & $\mathbf{4 , 4 4 3}$ & $\mathbf{4 , 4 6 8}$ & $\mathbf{4 , 3 6 3}$ & $\mathbf{4 , 5 2 9}$ & $\mathbf{4 , 5 6 2}$ & $\mathbf{4 , 3 7 4}$ \\
\hline • Turismo de sol y playa familiar. & 4,443 & 4,468 & 4,363 & 4,529 & 4,562 & 4,374 \\
\hline Turismo Deportivo: & $\mathbf{4 , 4 1 8}$ & $\mathbf{4 , 3 9 4}$ & $\mathbf{4 , 4 6 4}$ & $\mathbf{4 , 3 7 5}$ & $\mathbf{4 , 5 1 7}$ & $\mathbf{4 , 3 5 4}$ \\
\hline • Turismo Náutico. & 4,450 & 4,418 & 4,575 & 4,412 & 4,480 & 4,383 \\
\hline • Otras formas de turismo deportivo. & 4,440 & 4,426 & 4,425 & 4,412 & 4,562 & 4,393 \\
\hline • Cicloturismo. & 4,359 & 4,333 & 4,388 & 4,294 & 4,507 & 4,280 \\
\hline Turismo en la Naturaleza: & $\mathbf{4 , 5 4 9}$ & $\mathbf{4 , 5 6 2}$ & $\mathbf{4 , 6 1 7}$ & $\mathbf{4 , 4 1 9}$ & $\mathbf{4 , 5 5 3}$ & $\mathbf{4 , 5 0 0}$ \\
\hline - Turismo Rural. & 4,601 & 4,596 & 4,650 & 4,471 & 4,589 & 4,598 \\
\hline • Turísticos basados en la naturaleza. & 4,483 & 4,518 & 4,575 & 4,353 & 4,507 & 4,374 \\
\hline Turismo Cultural: & $\mathbf{4 , 4 4 3}$ & $\mathbf{4 , 4 6 8}$ & $\mathbf{4 , 3 6 3}$ & $\mathbf{4 , 5 2 9}$ & $\mathbf{4 , 5 6 2}$ & $\mathbf{4 , 3 7 4}$ \\
\hline $\begin{array}{l}\text { • Turismo basado en la cultura y } \\
\text { patrimonio. }\end{array}$ & 4,421 & 4,475 & 4,538 & 4,353 & 4,480 & 4,234 \\
\hline
\end{tabular}

Fuente: elaboración propia.

\section{RESULTADOS}

La comprobación de las hipótesis se realizó mediante Sistemas de Ecuaciones Estructurales (Gursoy et al., 2002; Lindberg \& Johnson, 1997), concretamente la regresión de Mínimos Cuadrados Parciales (Partial Least Squares - PLS). El software usado fue el SmartPLS 2.0M3 (Ringle et al., 2005). Aunque los resultados no difieren significativamente entre los distintos sistemas de ponderación alternativos, el más recomendado y usado en este caso es el path weighting, al proporcionar el valor de $\mathrm{R}^{2}$ más alto para las variables latentes (Henseler, Ringle \& Sinkovics, 2009). Antes de analizar el modelo estructural propuesto es necesario analizar el modelo de medida. Lo cual implica realizar una serie de comprobaciones para garantizar la calidad del modelo estructural: fiabilidad, validez convergente y validez discriminante.

Para comprobar la fiabilidad individual es necesario que los ítems usados posean cargas superiores a 0,707 (Carmines \& Zeller, 1979) y se comprobó que todos los ítems utilizados eran fiables (Tabla 4). La fiabilidad del constructo mide la consistencia de los indicadores. Los dos indicadores de fiabilidad más usados son el Alfa de Cronbach (Cronbach, 1970) y la fiabilidad compuesta (Werts, Linn \& Jöreskog, 1974). Al analizar los valores del Alfa de Cronbach para los constructos utilizados se obtienen valores buenos a excepción del constructo "Costes" donde es baja aunque asumible. La fiabilidad compuesta posee valores muy buenos en todos los caso (Tabla 3). Por tanto, podemos considerar que existe fiabilidad de los constructos utilizados. 
Tabla 3. Fiabilidad y validez convergente de los constructos.

\begin{tabular}{|l|c|c|c|c|c|}
\hline Constructo & AVE & $\begin{array}{c}\text { Fiabilidad } \\
\text { Compuesta }\end{array}$ & $\mathbf{R}^{\mathbf{2}}$ & $\begin{array}{c}\text { Alfa de } \\
\text { Cronbachs }\end{array}$ & Comunalidad \\
\hline Beneficios Económicos & 0,643 & 0,844 & 0,143 & 0,723 & 0,643 \\
\hline Otros Beneficios & 0,625 & 0,833 & 0,227 & 0,700 & 0,625 \\
\hline Costes & 0,749 & 0,856 & 0,140 & 0,665 & 0,749 \\
\hline Dependencia Regional & 1,000 & 1,000 & 0,000 & 1,000 & 1,000 \\
\hline Preocupación por el Medioambiente & 0,831 & 0,908 & 0,000 & 0,797 & 0,831 \\
\hline Actitud General hacia el Turismo & 0,589 & 0,852 & 0,385 & 0,768 & 0,589 \\
\hline Turismo de Sol y Playa & 1,000 & 1,000 & 0,084 & 1,000 & 1,000 \\
\hline Turismo Deportivo & 0,750 & 0,900 & 0,089 & 0,843 & 0,750 \\
\hline Turismo en la Naturaleza & 0,863 & 0,926 & 0,055 & 0,844 & 0,863 \\
\hline Turismo Cultural & 1,000 & 1,000 & 0,017 & 1,000 & 1,000 \\
\hline
\end{tabular}

Fuente: elaboración propia.

La validez convergente (Anderson \& Gerbing, 1988) implica que un conjunto de indicadores representa un único constructo subyacente, pudiendo ser esto demostrado por medio de su unidimensionalidad (Henseler et al., 2009) pero se suele valorar por medio de la Varianza Extraída Media (AVE) y, en este caso, los constructos utilizados muestran un AVE superior a 0,589 (Tabla 3 ) y superior al mínimo de 0,5 exigido por la literatura (Bagozzi \& Yi,
1988). La validez discriminante (Anderson \& Gerbing, 1988) implica que cada constructo debe ser diferente del resto. Al analizar las cargas cruzada y comparar las correlaciones con la raíz cuadrada de AVE (Chin, 1998) se confirmó que se cumple la validez discriminante. Tras el análisis del modelo de medida, las escalas utilizadas en el análisis del modelo estructural quedaron como aparecen en la Tabla 4.

Tabla 4. Medias, desviaciones y cargas de los ítems conservados.

\begin{tabular}{|l|c|c|c|}
\hline Denominación de la Variable Usada & $\begin{array}{c}\text { Media } \\
\text { aritmética }\end{array}$ & $\begin{array}{c}\text { Desviación } \\
\text { estándar }\end{array}$ & Carga \\
\hline Beneficios Económicos: & $\mathbf{4 , 3 7 4}$ & & \\
\hline$\bullet \quad$ Gracias al turismo las oportunidades de trabajo son más elevadas. & 4,495 & 0,657 & 0,818 \\
\hline $\begin{array}{l}\text { El turismo incrementa las oportunidades de negocio para los } \\
\text { residentes y las pequeñas empresas. }\end{array}$ & 4,325 & 0,748 & 0,804 \\
\hline $\begin{array}{l}\text { El turismo estimula que haya más empresas interesadas en invertir } \\
\text { en la isla. }\end{array}$ & 4,278 & 0,767 & 0,783 \\
\hline Otros Beneficios: & $\mathbf{3 , 2 2 8}$ & & 0,801 \\
\hline$\bullet \quad$ Gracias al turismo podemos tener más y mejores servicios públicos. & 3,478 & 1,182 & 0,797 \\
\hline $\begin{array}{l}\text { El turismo ha contribuido a que nuestros pueblos y ciudades estén } \\
\text { más cuidados y limpios. }\end{array}$ & 3,153 & 1,186 & 0,773 \\
\hline - La calidad de los servicios públicos es mejor gracias al turismo. & 3,048 & 1,165 & $\mathbf{3 , 0 0 0}$ \\
\hline Costes: & 2,770 & 1,214 & 0,882 \\
\hline $\begin{array}{l}\text { El turismo es la causa de la pérdida de nuestras costumbres y de } \\
\text { nuestra identidad. }\end{array}$ & 214 \\
\hline
\end{tabular}


Impacto de Las Actitudes de Los Residentes sobre El Grado de Aceptación Del Turismo de Playa, El Turismo de Deportes, El Turismo de Naturaleza Y El Turismo Cultural

\begin{tabular}{|c|c|c|c|}
\hline $\begin{array}{l}\text { - Debido al turismo debemos pagar unos impuestos y unas tasas más } \\
\text { elevadas. }\end{array}$ & 3,237 & 1,206 & 0,849 \\
\hline Dependencia Regional: & 4,644 & & \\
\hline $\begin{array}{l}\text { - Se ha de cuidar el turismo porque es básico para la economía de la } \\
\text { isla. }\end{array}$ & 4,644 & 0,652 & 1,000 \\
\hline Preocupación por el Medioambiente: & 3,779 & & \\
\hline $\begin{array}{l}\text { - El turismo ha contribuido a la degradación medioambiental de la } \\
\text { isla. }\end{array}$ & 3,713 & 1,161 & 0,926 \\
\hline $\begin{array}{l}\text { - El turismo ha provocado la saturación de algunos espacios } \\
\text { naturales. }\end{array}$ & 3,842 & 1,076 & 0,897 \\
\hline Actitud General hacia el Turismo: & 3,956 & & \\
\hline - Los efectos positivos superan a los negativos. & 3,660 & 1,021 & 0,776 \\
\hline - Creo que el turismo genera beneficios para todos. & 4,146 & 0,915 & 0,775 \\
\hline $\begin{array}{l}\text { - Estoy orgulloso de que tantos turistas quieran visitar y conocer } \\
\text { Ibiza. }\end{array}$ & 4,177 & 0,929 & 0,771 \\
\hline - Gracias al turismo tenemos una calidad de vida más elevada. & 3,799 & 0,992 & 0,749 \\
\hline Turismo de Sol y Playa: & $\mathbf{4 , 4 4 3}$ & & \\
\hline - Turismo de sol y playa familiar. & 4,443 & 0,808 & 1,000 \\
\hline Turismo Deportivo: & 4,418 & & \\
\hline - Turismo Náutico. & 4,450 & 0,818 & 0,887 \\
\hline - Otras formas de turismo deportivo. & 4,440 & 0,784 & 0,882 \\
\hline - Cicloturismo. & 4,359 & 0,819 & 0,829 \\
\hline Turismo en la Naturaleza: & 4,549 & & \\
\hline - Turismo Rural. & 4,600 & 0,671 & 0,948 \\
\hline - Turísticos basados en la naturaleza. & 4,483 & 0,833 & 0,909 \\
\hline Turismo Cultural: & 4,421 & & \\
\hline - Turismo basado en la cultura y el patrimon & 4,421 & 0,853 & 1,000 \\
\hline
\end{tabular}

Fuente: elaboración propia.

Una vez evaluado que el modelo de medida cumplía los criterios especificados se procedió al análisis del modelo estructural. Para contrastar si los coeficientes de regresión eran significativos fue necesario aplicar un procedimiento bootstrap no paramétrico (Davison \& Hinkley, 1997) con 5.000 submuestras creadas aleatoriamente a partir de la muestra original mediante sustitución con reemplazo. Las estimaciones de los parámetros generadas a partir de las submuestras se utilizaron para determinar los errores estándar de las estimaciones (Hair, Hult, Ringle \& Sarstedt, 2014) y los resultados obtenidos del análisis de significación se detallan en la Tabla 5.

Tabla 5. Coeficientes de regresión estandarizados y desviaciones.

\begin{tabular}{|l|c|c|c|c|}
\hline Relación Causal Analizada & $\begin{array}{c}\text { Valor } \\
\text { estimado }\end{array}$ & $\begin{array}{c}\text { Error } \\
\text { estándar }\end{array}$ & $\begin{array}{c}\text { t de } \\
\text { Student }\end{array}$ & P valor \\
\hline B. Económicos $\rightarrow$ Act. General (Hip. 1) & $0,283^{* *}$ & 0,103 & 2,760 & 0,003 \\
\hline Otr. Beneficios $\rightarrow$ Act. General (Hip. 2) & $0,292^{* *}$ & 0,102 & 2,872 & 0,002 \\
\hline B. Económicos $\rightarrow$ Otr. Beneficios (Hip. 3) & $0,261^{* *}$ & 0,103 & 2,525 & 0,006 \\
\hline Costes $\rightarrow$ Act. General (Hip. 4) & $-0,096^{\mathrm{ns}}$ & 0,091 & 1,055 & 0,146 \\
\hline Dep. Regional $\rightarrow$ B. Económicos (Hip. 5.1) & $0,360^{* *}$ & 0,129 & 2,788 & 0,003 \\
\hline Dep. Regional $\rightarrow$ Otr. Beneficios (Hip. 5.2) & $0,137^{\mathrm{ns}}$ & 0,132 & 1,040 & 0,149 \\
\hline Dep. Regional $\rightarrow$ Costes (Hip. 5.3) & $-0,079^{\mathrm{ns}}$ & 0,100 & 0,790 & 0,215 \\
\hline
\end{tabular}


Impacto de Las Actitudes de Los Residentes sobre El Grado de Aceptación Del Turismo de Playa, El Turismo de Deportes, El Turismo de Naturaleza Y El Turismo Cultural

\begin{tabular}{|l|c|c|c|c|}
\hline \hline Dep. Regional $\rightarrow$ Act. General (Hip. 5.4) & $0,200^{*}$ & 0,108 & 1,851 & 0,032 \\
\hline Pre. Medioamb. $\rightarrow$ B. Económicos (Hip. 6.1) & $-0,098^{\text {ns }}$ & 0,092 & 1,068 & 0,143 \\
\hline Pre. Medioamb. $\rightarrow$ Otr. Beneficios (Hip. 6.2) & $-0,303^{* * *}$ & 0,091 & 3,349 & 0,000 \\
\hline Pre. Medioamb. $\rightarrow$ Costes (Hip. 6.3) & $0,362^{* * *}$ & 0,091 & 3,981 & 0,000 \\
\hline Pre. Medioamb. $\rightarrow$ Act. General (Hip. 6.4) & $-0,064^{\text {ns }}$ & 0,089 & 0,720 & 0,236 \\
\hline Act. General $\rightarrow$ Tur. Sol y Playa (Hip. 7.1) & $0,289^{* *}$ & 0,115 & 2,513 & 0,006 \\
\hline Act. General $\rightarrow$ Tur. Deportivo (Hip. 7.2) & $0,298^{* *}$ & 0,106 & 2,810 & 0,002 \\
\hline Act. General $\rightarrow$ Tur. Naturaleza (Hip. 7.3) & $0,235^{*}$ & 0,118 & 1,999 & 0,023 \\
\hline Act. General $\rightarrow$ Tur. Cultural (Hip. 7.4) & $0,130^{\text {ns }}$ & 0,107 & 1,218 & 0,112 \\
\hline
\end{tabular}

* Significativo al 0’05; ** Significativo al 0’01; *** Significativo al 0’001; ns No significativo.

Fuente: elaboración propia.

En base a los resultados obtenidos (Tabla 5), el efecto de la percepción de los beneficios económicos y de los otros beneficios sobre la actitud general hacia el turismo es positivo y significativo, tal y como se planteaba en las Hipótesis 1 y 2. Cabe indicar que existe un efecto positivo y significativo de los beneficios económicos sobre los otros beneficios, tal y como proponía la Hipótesis 3. El efecto de los costes sobre la actitud general hacia el turismo es negativo pero no significativo $\mathrm{y}$, por tanto no puede aceptarse la Hipótesis 4. La percepción de que Ibiza depende del sector turístico posee un efecto positivo y significativo sobre la percepción de los beneficios económicos (Hipótesis 5.1) y sobre la actitud general hacia el turismo (Hipótesis 5.4), pero no posee un efecto significativo sobre otros beneficios (Hipótesis 5.2) y costes (Hipótesis 5.3). Como indica la bibliografía consultada (Besculides et al., 2002; Gursoy et al., 2002; Haralambopoulos \& Pizam, 1996; Liu \& Var, 1986; Williams \& Lawson, 2001), la dependencia económica regional aumenta la valoración de los beneficios económicos. Por otra parte, la preocupación por el medioambiente posee un efecto negativo muy significativo sobre los otros beneficios (Hipótesis 6.2) y positivo sobre los costes (Hipótesis 6.3) pero no sobre los beneficios económicos (Hipótesis 6.1) o la actitud general hacia el turismo, siendo resultados parecidos a los de Gursoy et al. (2002).

Finalmente, cabe indicar que la actitud general de los residentes hacia el turismo tiene efectos significativos y positivos (Tabla 5) sobre el turismo de sol y playa (Hipótesis 7.1), el turismo deportivo (Hipótesis 7.2) y el turismo de naturaleza (Hipótesis 7.3), pero no hay un efecto significativo sobre el turismo cultural (Hipótesis 7.4). De ellos, los dos efectos más importantes $(\beta>0,250)$ y significativos $(\mathrm{P}>0,01)$ son sobre el turismo de sol y playa y el turismo deportivo. Al llegar a este punto se planteó que sucedería si se eliminara el constructo intermedio de "Actitud General hacia el Turismo" y se plantearan relaciones causales directas de los constructos "Beneficios Económicos", "Otros Beneficios", "Costes", "Dependencia Regional" y "Preocupación por el Medioambiente" con los constructos "Turismo de Sol y Playa", "Turismo Deportivo", "Turismo en la Naturaleza" y "Turismo Cultural". Los resultados de este análisis aparecen en la Tabla 6.

Tabla 6. Coeficientes de regresión estandarizados y desviaciones.

\begin{tabular}{|l|c|c|c|c|}
\hline Relación Causal Analizada & $\begin{array}{c}\text { Valor } \\
\text { estimado }\end{array}$ & $\begin{array}{c}\text { Error } \\
\text { estándar }\end{array}$ & $\begin{array}{c}\text { t de } \\
\text { Student }\end{array}$ & P value \\
\hline B. Económicos $\rightarrow$ Otr. Beneficios (Hip. 3) & $0,262 * *$ & 0,104 & 2,521 & 0,006 \\
\hline
\end{tabular}


Impacto de Las Actitudes de Los Residentes sobre El Grado de Aceptación Del Turismo de Playa, El Turismo de Deportes, El Turismo de Naturaleza Y El Turismo Cultural

\begin{tabular}{|c|c|c|c|c|}
\hline Dep. Regional $\rightarrow$ B. Económicos (Hip. 5.1) & $0,360^{* *}$ & 0,128 & 2,820 & 0,002 \\
\hline Dep. Regional $\rightarrow$ Otr. Beneficios (Hip. 5.2) & $0,139^{\mathrm{ns}}$ & 0,130 & 1,067 & 0,143 \\
\hline Dep. Regional $\rightarrow$ Costes (Hip. 5.3) & $-0,080^{\mathrm{ns}}$ & 0,099 & 0,808 & 0,210 \\
\hline Pre. Medioamb. $\rightarrow$ B. Económicos (Hip. 6.1) & $-0,099^{\mathrm{ns}}$ & 0,095 & 1,044 & 0,148 \\
\hline Pre. Medioamb. $\rightarrow$ Otr. Beneficios (Hip. 6.2) & $-0,301 * * *$ & 0,095 & 3,157 & 0,001 \\
\hline Pre. Medioamb. $\rightarrow$ Costes (Hip. 6.3) & $0,363 * * *$ & 0,092 & 3,937 & 0,000 \\
\hline \multicolumn{5}{|l|}{ Nuevas Relacionas Causales: } \\
\hline B. Económicos $\rightarrow$ Tur. Sol y Playa & $0,120^{\mathrm{ns}}$ & 0,127 & 0,939 & 0,174 \\
\hline B. Económicos $\rightarrow$ Tur. Deportivo & $0,166^{\mathrm{ns}}$ & 0,130 & 1,273 & 0,102 \\
\hline B. Económicos $\rightarrow$ Tur. Naturaleza & $0,133^{\mathrm{ns}}$ & 0,126 & 1,053 & 0,146 \\
\hline B. Económicos $\rightarrow$ Tur. Cultural & $0,150^{\phi}$ & 0,115 & 1,308 & 0,095 \\
\hline Otr. Beneficios $\rightarrow$ Tur. Sol y Playa & $-0,033^{\mathrm{ns}}$ & 0,114 & 0,292 & 0,385 \\
\hline Otr. Beneficios $\rightarrow$ Tur. Deportivo & $-0,086^{\mathrm{ns}}$ & 0,127 & 0,675 & 0,250 \\
\hline Otr. Beneficios $\rightarrow$ Tur. Naturaleza & $-0,078^{\mathrm{ns}}$ & 0,123 & 0,634 & 0,263 \\
\hline Otr. Beneficios $\rightarrow$ Tur. Cultural & $-0,107^{\mathrm{ns}}$ & 0,116 & 0,917 & 0,180 \\
\hline Costes $\rightarrow$ Tur. Sol y Playa & $-0,107^{\mathrm{ns}}$ & 0,114 & 0,933 & 0,175 \\
\hline Costes $\rightarrow$ Tur. Deportivo & $-0,059^{\text {ns }}$ & 0,121 & 0,492 & 0,312 \\
\hline Costes $\rightarrow$ Tur. Naturaleza & $-0,086^{\mathrm{ns}}$ & 0,117 & 0,734 & 0,232 \\
\hline Costes $\rightarrow$ Tur. Cultural & $-0,079^{\mathrm{ns}}$ & 0,110 & 0,720 & 0,236 \\
\hline Dep. Regional $\rightarrow$ Tur. Sol y Playa & $0,209 *$ & 0,124 & 1,681 & 0,046 \\
\hline Dep. Regional $\rightarrow$ Tur. Deportivo & $0,207^{*}$ & 0,116 & 1,790 & 0,037 \\
\hline Dep. Regional $\rightarrow$ Tur. Naturaleza & $0,144^{\mathrm{ns}}$ & 0,113 & 1,274 & 0,101 \\
\hline Dep. Regional $\rightarrow$ Tur. Cultural & $0,118^{\mathrm{ns}}$ & 0,107 & 1,110 & 0,134 \\
\hline Pre. Medioamb. $\rightarrow$ Tur. Cultural & $0,015^{\mathrm{ns}}$ & 0,110 & 0,133 & 0,447 \\
\hline Pre. Medioamb. $\rightarrow$ Tur. Deportivo & $0,065^{\mathrm{ns}}$ & 0,124 & 0,524 & 0,300 \\
\hline Pre. Medioamb. $\rightarrow$ Tur. Naturaleza & $0,045^{\mathrm{ns}}$ & 0,113 & 0,397 & 0,346 \\
\hline Pre. Medioamb. $\rightarrow$ Tur. Sol y Playa & $0,009^{\mathrm{ns}}$ & 0,117 & 0,077 & 0,469 \\
\hline
\end{tabular}

Fuente: elaboración propia.

Este segundo análisis (Tabla 6) muestra que las relaciones causales conservadas del modelo propuesto inicialmente (Figura 1) no varían ni en valor ni en significación, pero las nuevas relaciones causales no resultan significativas en la mayoría de los casos (Tabla 6):

- La percepción de los beneficios económicos sólo muestra un efecto positivo y significativo al 0,1 en el caso del turismo Cultural. Este valor tan poco significativo hace dudar de que realmente pueda existir esta relación causal.

- La dependencia regional del turismo sólo tiene efectos significativos en el caso del turismo de sol y playa y el turismo deportivo. En ambos casos poseen valores estimados superiores a 0,200 y son significativos al 0,05 . Llama la atención que el turismo deportivo posea un comportamiento similar en este estudio al de sol y playa.

- La percepción de los otros beneficios, la percepción de los costes y la preocupación por el medioambiente no tienen efectos significativos sobre ninguno de los cuatro tipos de turismo tomados en consideración.

Los resultados obtenidos muestra que era más adecuado el modelo causal planteado en un primer momento (Figura 1), en el cual el grado de aceptación de los diversos tipos de turismo depende de la actitud general 
hacia el turismo. Cabe indicar que si se analizan los efectos totales del modelo propuesto (Tabla 7) se observa que los efectos indirectos sobre los tipos de turismo son muy bajos, siendo los dos mayores valores los efectos de la dependencia económica sobre el turismo de sol y playa $(0,109)$ y el turismo deportivo $(0,112)$, únicas relaciones causales significativas al 0,05 en el segundo análisis. El efecto indirecto de los beneficios económicos sobre el turismo de sol y playa $(0,104)$ y el turismo deportivo $(0,107)$ posee valores cercanos a los de la dependencia económica sobre esos mismos tipos de turismo, pero en el segundo análisis no resultan significativas las correspondientes relaciones causales. Posiblemente haya que poner en duda la significación del efecto directo de la dependencia económica sobre el turismo de sol y playa y el turismo deportivo.

Tabla 7. Efectos totales del modelo causal propuesto.

\begin{tabular}{|l|c|c|c|c|c|c|}
\hline \multirow{2}{*}{$\begin{array}{l}\text { Variables } \\
\text { Causales }\end{array}$} & \multirow{2}{*}{$\begin{array}{l}\text { Actitud } \\
\text { General }\end{array}$} & \multicolumn{5}{|c|}{ Efectos sobre el tipo de turismo } \\
\cline { 3 - 7 } & & Efecto & Sol y Playa & Deportivo & Naturaleza & Cultural \\
\hline Act. General & - & Directo & 0,289 & 0,298 & 0,235 & 0,130 \\
\hline B. Económicos & 0,359 & Indirecto & 0,104 & 0,107 & 0,085 & 0,047 \\
\hline Otr. Beneficios & 0,292 & Indirecto & 0,084 & 0,087 & 0,069 & 0,038 \\
\hline Costes & $-0,096$ & Indirecto & $-0,028$ & $-0,029$ & $-0,023$ & $-0,013$ \\
\hline Dep. Regional & 0,377 & Indirecto & 0,109 & 0,112 & 0,089 & 0,049 \\
\hline Pre. Medioamb. & $-0,222$ & Indirecto & $-0,064$ & $-0,066$ & $-0,052$ & $-0,029$ \\
\hline
\end{tabular}

Fuente: elaboración propia.

\section{CONCLUSIONES}

Primeramente hay que indicar que la dependencia económica tiene efectos sobre la percepción de los impactos del turismo, valorando de forma más positiva los beneficios económicos y el turismo en su conjunto. La preocupación por el medioambiental posee efectos importantes sobre los otros beneficios, en sentido negativo, y los costes, en sentido negativo. Los beneficios y la dependencia económica regional son fundamentales para determinar la actitud general de los residentes hacia el turismo.

Al analizar el efecto de la actitud de los residentes sobre el grado de aceptación de las distintas ofertas turísticas se observa que no hay efectos significativos sobre el turismo cultural. En los otros tres tipos de turismo las relaciones son significativas, aunque los efectos son más importantes y significativas en el caso del turismo deportivo y el turismo de sol y playa, el cual actúa de referencia al tratarse del mayoritario en la isla. En los efectos indirectos y en el segundo análisis hay pocos resultados significativos pero estos asemejan el turismo deportivo al turismo de sol y playa familiar. Posiblemente sea un indicio de que el turismo deportivo es el mejor plan alternativo para la diversificación del actual turismo de sol y playa. Los otros dos tipos de turismo poseen elevados grados de aceptación por parte de los residentes pero es una respuesta con poca (turismo de naturaleza) o ninguna relación (turismo cultural) con el proceso de análisis del turismo que realizan los residentes. Posiblemente sea una respuesta más "automática" y menos causada por un análisis crítico del turismo.

En base a los resultados de este estudio parece razonable priorizar la potenciación de deportes que aprovechen las infraestructuras actualmente existentes en la 
isla y que posean la capacidad de atraer turistas. Aunque el grado de aceptación del turismo deportivo no sea el más elevado, su mayor relación con la percepción de depender económicamente del turismo y con la percepción de los beneficios económicos hace posible que en situaciones de crisis económica, o cuando ya genere claros beneficios económicos, este turismo mejore su grado de aceptación más que otras ofertas. En los últimos años se ha potenciado la celebración de eventos deportivos en Ibiza, especialmente competiciones náuticas como el Ibiza Mediterranean Grand Prix, que engloba pruebas oficiales del campeonato mundial UIM CLASS-1 World Powerboat Championship y del UIM-ABP Aquabike Class Pro World Championship.

\section{REFERENCIAS}

Agència de Turisme de les Illes Balears (2015). El turisme a les Illes Balears: Anuari 2014. Palma de Mallorca: Agència de Turisme de les Illes Balears.

Akis, S., Peristianis, N., \& Warner, J. (1996). Residents' attitudes to tourism development: the case of Cyprus. Tourism Management, 17(7), 481-494.

Allen, L. R., Hafer, H. R., Long, P. T., \& Perdue, R. R. (1993). Rural residents' attitudes toward recreation and tourism development. Journal of Travel Research, 32(4), 27-33.

Almeida, F., Balbuena, A., \& Cortés, R. (2015). Resident's attitudes towards the impacts of tourism. Tourism Management Perspectives, 13, 33-40.

Andereck, K. L., Valentine, K. M., Knoff, R. C., \& Vogt, C. A. (2005). Residents' perceptions of community
En futuros estudios se deberá repetir el análisis con nuevos tipos de turismo para intentar determinar cuando la aceptación de un tipo de oferta turística es el resultado de su actitud hacia el turismo o una respuesta inconexa $\mathrm{y}$, posiblemente, no meditada. También es necesario analizar los distintos subtipos de turismo, por ejemplo los diversos tipos de turismo deportivo. Cabe indicar que las opiniones en los medios de comunicación locales son muy distintas en relación del cicloturismo y al turismo de golf. En el primer caso hay un apoyo mayoritario y en el segundo caso las críticas son mayoritarias. Por tanto, los resultados del cicloturismo no son aplicables al golf ni los resultados del golf son aplicables al cicloturismo.

tourism impacts. Annals of Tourism Research, 32(4), 1056-1076.

Anderson, J. C., \& Gerbing, D. W. (1988). Structural equation modeling in practice: A review and recommended twostep approach. Psychological Bulletin, 103, 411-423.

Ap, J. (1992). Residents' perceptions on tourism impacts . Annals of Tourism Research, 19(4), 665-690.

Ap, J., \& Crompton, J. L. (1998). Developing and testing a tourism impact scale. Journal of Travel Research, 37(2), 120-130.

Ayres, R. (2000). Tourism as a passport to development in small states: Reflections on Cyprus. International Journal of Social Economics, 27(2), 114133. 
Bagozzi, R. P., \& Yi, Y. (1988). On the evaluation of structural equation models. Journal of the Academy of Marketing Science, 16(2), 74-94.

Besculides, A., Lee, M., \& McCormick, P. (2002). Resident's perceptions of the cultural benefits of tourism. Annals of Tourism Research, 29(2), 303-319.

Bigné, J. E., Font, X., \& Andreu, L. (2000). Marketing de destinos turísticos. Análisis y estrategias de desarrollo. Madrid: Editorial ESIC.

Bramwell, B. (2003). Maltese responses to tourism. Annals of Tourism Research, 30(3), 581-605.

Brayley, R., Var, T., \& Sheldon, P. (1990). Perceived influence of tourism on social issues. Annals of Tourism Research, 17(2), 285-289.

Bruckner, P., \& Finkielkraut, A. (1979). Au Coin de la Rue. L'aventure. Paris: Èditions du Seuil.

Bruner, E. (1996). Tourism in Ghana: the representation of slavery and the return of the black diaspora. American Anthropologist, 98(2), 290-304.

Bujosa, A., \& Rosselló, J. (2007). Modelling environmental attitudes toward tourism. Tourism Management, 28(3), 688695.

Carmines, E., \& Zeller, R. (1979). Reliability and validity assessment, N. 07017. Sage University Paper Series on Quantitative Applications in the Social Sciences. Beverly Hills, CA: Sage.

Cater, E. A. (1987). Tourism in the least developed countries. Annals of Tourism Research 14(2), 202-226.
Chin, W. W. (1998). Issues and opinions on structural equation modeling. MIS Quarterly, 22(1), 7-16.

Cronbach, L. J. (1970). Essentials of Psychological Testing. 3rd ed. New York, NY: Harper \& Row.

Davison, A., \& Hinkley, D. (1997). Bootstrap Methods and Their Application. Cambridge: Cambridge University Press.

Doran, R., Larsen, S., \& Wolff, K. (2015). Different but similar: Social comparison of travel motives among tourists. International Journal of Tourism Research, 17(6), 555-563.

Faulkner, B., \& Tideswell, C. A. (1997). Framework for monitoring community impacts of tourism. Journal of Sustainable Tourism, 5(1), 3-28.

Garau, J. B. (2002). Agroturismo y turismo rural en Balears. Palma de Mallorca: Edicions CAEB.

Garau, J. B., Serra, A., \& Tur, V. (1997). El turismo de Golf en Balears. Palma de Mallorca: Edicions CAEB.

Garau, J. B., Serra, A., \& Tur, V. (1999a). El cicloturismo en Baleares. Palma de Mallorca: Edicions CAEB.

Garau, J. B., Serra, A., \& Tur, V. (1999b). El turismo activo en Baleares. Palma de Mallorca: Edicions CAEB.

Gee, C. Y., Mackens, J. C., \& Choy, D. J. (1989). The Travel Industry. New York, NY: Van Nostrand Reinhold.

Gómez, L., San Martín, J., \& Bertiche, C. (1999). A model of tourism experience and attitude change. Annals of Tourism Research, 26(4), 1024-1027. 
Gration, D., Raciti, M., \& Arcodia, C. (2011). The role of consumer self-concept in marketing festivals. Journal of Travel and Tourism Marketing, 28(6), 644-655.

Gursoy, D., \& Kendall, K. W. (2006). Hosting mega events: modeling locals' support. Annals of Tourism Research, $33(3), 603-623$.

Gursoy, D., \& Rutherford, D. (2004). Host atttitudes toward tourism: An Improved Structtural Model. Annals of Tourism Research, 31(3), 495-516.

Gursoy, D., Jurowski, C., \& Uysal, M. (2002). Resident attitudes: a structural Modeling Approach. Annals of Tourism Research, 29(1), 79-105.

Gustafson, P. (2002). Tourism and seasonal retirement migration. Annals of Tourism Research, 29(4), 899-918.

Hair, J., Hult, G., Ringle, C., \& Sarstedt, M. (2014). A Primer on Partial Least Squares Structural Equation Modeling (PLS-SEM). Thousand Oaks, CA: Sage.

Haralambopoulos, N., \& Pizam, A. (1996). Perceived impacts of tourism: the case of Samos. Annals of Tourism Research, 23(3), 503-526.

Hennig, C. (1997). Touristenbeschimpfung. Zur geschichte des anti-tourismus. Zeitschrift für Volkskunde, 93(1), 31.41.

Henseler, J., Ringle, C. M. and Sinkovics, R. R. (2009). The use of Partial Least Squares Path Modeling in international marketing. En Sinkovics, R.R. and Ghauri, P.N. (Ed.), Advances in

International Marketing, Emerald, Bingley, WA, pp. 277-320.
Institut d'Estadística de les Illes Balears (Ibestat). Consultado el 15 de marzo de 2015 http://ibestat.caib.es/

Jacobsen, J. K. S. (2000). Anti-tourist attitudes: mediterranean charter tourism. Annals of Tourism Research, 27(2), 284300.

Johnson, J. D., Snepenger, D. J., \& Akis, S. (1994). Residents' perceptions of tourism development. Annals of Tourism Research, 21(3), 629-642.

Jurowski, C., Uysal, M., \& Williams, R. D. (1997). A theoretical analysis of host community resident reactions to tourism. Journal of Travel Research, 36(2), 3-11.

Keogh, B. (1990). Resident and recreationists' perceptions and attitudes with respect to tourism development. Journal of Applied Recreation Research, 15(2), 71-83.

Kim, S. S., \& Petrick, J. F. (2005). Residents' perceptions on impacts of the FIFA 2002 World Cup: the case of Seoul as a host city. Tourism Management, 26(1), 25-38.

King, B., Pizam, A., \& Milman, A. (1993). Social impacts of tourism: Host perceptions. Annals of Tourism Research, 20(4), 650-665.

Ko, D. W., \& Stewart, W. P. (2002). A structural equation model of residents' attitudes for tourism development. Tourism Management, 23(5), 521-530.

Lankford, S. V., \& Howard, D. R. (1994). Developing a tourism impacts

attitude scale. Annals of Tourism Research, 21(1), 121-139. 
Lanquar, R. (2001). Marketing turístico: de lo global a lo local. Barcelona: Editorial Ariel.

Lawson, R. W., Williams, J., Young, T., \& Cossens, J. (1998). A comparison of residents attitudes towards tourism in 10 New Zealand destinations. Tourism Management, 19(3), 247-256.

Lee, C. K., \& Back, K. J. (2006). Examining structural relationships among perceived impact, benefit, and support for casino development based on 4 year longitudinal data. Tourism Management, 27(3), 466-480.

Lee, C. K., Kim, S. S., \& Kang, S. (2003). Perceptions of casino impacts: A Korean longitudinal study. Tourism Management, 24(1), 45-55.

Lepp, A. (2007). Residents' attitudes towards tourism in Bigodi village, Uganda. Tourism Management, 28(3), 876-885.

Lindberg, K., \& Johnson, R. L. (1997). Modeling residents attitudes toward tourism. Annals of Tourism Research, 24(2), 402-424.

Lindberg, K., Dellaert, B. G. C., \& Rassing, C. R. (1999). Resident tradeoffs. A choise modeling approach. Annals of Tourism Research, 26(3), 554-569.

Liu, J. C., \& Var, T. (1986). Residents attitudes toward tourism impacts in Hawaii. Annals of Tourism Research, 13(2), 193214.

Liu, J. C., Sheldon, P. J., \& Var, T. (1987). Residents perceptions of the environmental impacts of tourism. Annals of Tourism Research, 14(1), 17-37.

Long, P. T., Perdue, R.R., \& Allen, L. (1990). Rural resident tourism perceptions and attitudes by community level of tourism. Journal of Travel Research, 28(3), 3-9.

Mackay, K. J., \& Campbell, J. M. (2004). An examination of residents' support for hunting as a tourism product. Tourism Management, 25(4), 443-452.

Madrigal, R. (1993). A tale of tourism in two cities. Annals of Tourism Research, 20(2), 336-353.

Mason, P., \& Cheyne, J. (2000). Resident's attitudes to proposed tourism development. Annals of Tourism Research, 27(2), 391-411.

McIntosh, R. W., \& Goeldner, C.R. (1990). Tourism Principles, Practices, Philosophies. New York, NY: Wiley.

Milman, A., \& Pizam, A. (1988). Social impact of tourism on Central Florida. Annals of Tourism Research, 15(2), 191204.

Murphy, P. E. (1985). Tourism: A community approach. New York, NY: Routledge.

Nyaupane, G. P., Teye, V., \& Paris, C. (2008). Innocents abroad. attitude change toward hosts. Annals of Tourism Research, 35(3), 650-667.

Pearce, D. G. (1996). Tourist organizations in Sweden. Tourism Management, 17(6), 413-424.

Perdue, R. R., Long, P. T., \& Allen, L. (1987). Rural resident tourism perceptions and attitudes. Annals of Tourism Research, 14(3), 420-429.

Perdue, R. R., Long, P. T., \& Kang, Y. S. (1995). Resident support for gambling as 
a tourism development strategy. Journal of Travel Research, 34(2), 3-11.

Plog, S. C. (1974). Why destination areas rise and fall in popularity. Cornell Hotel and Restaurant Administration Quarterly, 14(4), 55-58.

Plog, S. C. (2002). The power of psychographics and the concept of venturesomeness. Journal of Travel Research, 40(3), 244-251.

Prentice, R. (1993). Communitydriven tourism planning and residents' preferences. Tourism Management, 14(3), 218-227.

Ramón, J. (2015). Efecto de la economía y el entorno en los residentes. PASOS: Revista de Turismo y Patrimonio Cultural, 13(6), 1371-1386.

Ramón, J., Azpelicueta, M. C., \& Serra, A. (2015). 'Ibiza, Biodiversidad y Cultura': Evolución y potencial turístico de un emplazamiento patrimonio mundial. International Journal of Scientific Management and Tourism, 1(1), 109-133.

Ringle, C. M., Wende, S., \& Will, S. (2005). SmartPLS 2.0 (Beta). Hamburg: SmartPLS.

Santesmases, M. (2009). DYANE Versión 4: Diseño y análisis de encuestas en investigación social y de mercados. Madrid: Ediciones Pirámide.

Serra, A., Ramón, J., \& Estades, R. (2015). Characteristics and peculiarities of rural tourism in the Balearic Islands. Tourism Planning \& Development, 12(2), 125-144.
Sharpley, R. (2014). Host perceptions of tourism: A review of the research. Tourism Management, 42, 37-49.

Teye, V., \& Sirakaya, E., \& Sönmez, S. F. (2002). Resident's attitudes toward tourism development. Annals of Tourism Research, 29(3), 668-688.

Um, S., \& Crompton, J. L. (1987). Measuring resident's attachment levels in a host community. Journal of Travel Research, 26(1), 27-29.

Var, T., Kendall, K. W., \& Tarakcoglu, E. (1985). Residents attitudes toward tourists in a turkish resort town. Annals of Tourism Research, 12(4), 652-658.

Vong, F. (2009). Changes in residents' gambling attitudes and perceived impacts at the fifth anniversary of Macao's gaming deregulation. Journal of Travel Research, 47(3), 388-397.

Vong, F., \& McCartney, G. (2005). Mapping resident perceptions of gaming impact. Journal of Travel Research, 44(2), 177-187.

Weaver, D. B., \& Lawton, L. J. (2001). Resident perceptions in the urban-rural frige. Annals of Tourism Research, 28(2), 439-458.

Werts, C. E., Linn, R. L., \& Jöreskog, K. G. (1974). Interclass reliability estimates: testing structural assumptions. Educational and Psychological Measurement, 34, 25-33.

Williams, J., \& Lawson, R. (2001). Community issues and resident opinions of tourism. Annals of Tourism Research, 28(2), 269-290. 
Yu, J., \& Lee, T. J. (2014). Impact of tourists' intercultural interactions. Journal of Travel Research, 53(2), 225-238.

Yu, X., Kim, N., Chen, C., \& Schwartz, Z. (2012). Are you a tourist? tourism definition from the tourist perspective. Tourism Analysis, 17(4), 445457. 